\title{
Cross-modality matching functions generated by magnitude estimation
}

\author{
JOSEPH C. STEVENS and LAWRENCE E. MARKS \\ John B. Pierce Foundation Laboratory, New Haven, Connecticut 06519 \\ and Yale University, New Haven, Connecticut 06520
}

\begin{abstract}
It is possible to generate cross-modality matching functions by having subjects make magnitude estimates of sets of stimuli appropriate to different modalities. The sets are interspersed among each other in the same test session and judged on a common absolute scale of sensory magnitude. An appropriate statistical device locates stimulus levels that appear, on the average, to match. The method is fast, efficient, circumvents the need for continuous stimulus adjustment, and holds promise for the study of the individual as well as the average psychophysical function. To illustrate its potential uses, advantages, and limitations, we used the method to generate cross-modality matching functions relating loudness and brightness. Compared to the scales of loudness and brightness generated by the magnitude estimations of the same stimuli, the matching functions (1) conform better to power functions, (2) may show less variation in slope (exponent), and (3) show far less variation in absolute mag. nitude (position).
\end{abstract}

This paper describes a simple procedure for generating cross-modality matching functions that has several advantages over the usual method of matching by continuous stimulus adjustment. The procedure promises to be useful in the comparison of groups of subjects (e.g., normal vs. pathological sensory modalities) and for the assessment of sensory functioning in the individual subject. We call the method "magnitude matching."

The method calls upon the subject to make magnitude estimations of sets of stimuli appropriate to two or more sense modalities, alternating from one modality to another from trial to trial, under instruction to estimate all presentations on a common scale of sensory magnitude. The rationale for this method is that when a person, on the average, assigns the same number to two different sensory events, he means to say that they have the same sensory magnitude. The magnitude estimations are statistically processed to generate pairs of stimulus values that appear to match. This paper illustrates the use of magnitude matching applied to loudness and brightness.

\section{Conventional Cross-Modality Matching}

In the typical cross-modality matching study, the subject adjusts the level of modality A to match various levels of modality B. To minimize the effects of regression bias (S. S. Stevens \& Greenbaum, 1966), it is advisable to reverse the experiment and to match

Requests for reprints may be addressed to Joseph C. Stevens, John B. Pierce Foundation Laboratory, 290 Congress Avenue, New Haven, Connecticut 06519.
B to $A$ as well, or to obtain matches also to a third modality, C (Cross, Tursky, \& Lodge, 1975).

Although the method has been widely used and was important in the development of the power law of sensory magnitude (S. S. Stevens, 1975), its applicability is limited (for a summary, see S. S. Stevens, 1975; for studies of brightness and loudness, see J. C. Stevens, 1957, and J. C. Stevens \& Marks, 1965). For one thing, there are modalities, such as olfaction, taste, and common chemical sensation, for which continuous stimulus adjustment is impractical. Also, the method is awkward for continua that exhibit fast adaptation, such as brightness, thermal sensation, taste, and olfaction. To get a good match, the stimuli must be sampled frequently, but to maintain adaptation level, the samplings should be separated by long periods of rest. This taxes the memory of the subject and prolongs the experiment.

\section{Advantages of the New Method}

The method of magnitude matching helps to solve these and other problems. Compared to conventional cross-modality matching, it has the following advantages: (1) It is free of the problem of regression bias, so it is unnecessary to do the experiment two ways. (2) It is applicable to all continua, including those for which continuous variation is impractical. (3) Only brief, simple stimulus observation is required, which helps control the problem of adaptation. (4) The experiment takes much less time and is easier on both the subject and the experimenter. This speed permits the generation of matching functions from individual subjects in a single brief session. (5) The apparatus needed is far simpler, since only fixed steps 
on the stimulus dimension are needed. This simplicity makes experiments feasible in laboratories that might otherwise have to forego them. (6) The method may prove valuable for comparison among individuals or between groups of individuals with regard to sensory magnitude. In fact, the present study grew out of aspirations in our laboratory to evaluate the effects on suprathreshold magnitude of such factors as age, sex, and sensory pathology. Studies that proceed naively to apply the method of magnitude estimation to such problems often overlook large individual idiosyncrasies in the use of the number scale to represent magnitudes and are subject to potentially devastating biases. For example, the comparison of groups of old and young subjects may reveal differences that stem from cognitive, as opposed to organic, differences between the old and the young, and such cognitive differences could stem from mental changes with age or from differences in educational levels.

\section{Theoretical Basis of the New Method}

There are three ways in which personal number behavior can affect magnitude estimations-(1) in the relative range of numbers the subject assigns to a set of stimuli, reflecting itself in the slope of a function; (2) in the absolute size of the numbers the subject assigns to the set, reflecting itself in the intercept (up-down position) of the function; and (3) in the mathematical form of the function, reflecting itself in curvature of the person's function when plotted in $\log$-log coordinates. The rationale behind magnitude matching is that personal numerical idiosyncrasies that the subject brings to the task will tend to apply to all the stimuli under judgment in the test session, and their effects, therefore, should tend to cancel out of the matching function. In this sense, a subject can serve as his or her own control and thereby reduce variability in slope, position, and shape. Thus, potential biases associated with the comparison of groups of subjects selected on the basis of some trait or other can be averted.

This rationale for magnitude matching rests both on casual observation of the "number behavior" of hundreds of subjects over the years and also on experimental study of individual variation, especially in the exponent, of the power function. In perhaps the first study of the matter, Jones and Marcus (1961) demonstrated significant correlations among the sizes of exponents for three different modalities studied in the same test session. Similar results were reported by Ekman, Hosman, Lindman, Ljungberg, \& Åkesson (1968) and Jones and Woskow (1962). M. Teghtsoonian and $R$. Teghtsoonian (1971) carried the matter a step farther by examining the correlations among exponents obtained by individual subjects in repeated tests over time. The correlation between two tests approached zero as the time between them became sufficiently long.
These findings suggest that the individual differences in exponent probably stem primarily from personal judgmental differences. Real sensory differences may exist, of course-surely they do in frank pathology, such as deafness-but the large personal judgmental factor makes them all the harder to detect with confidence. The method of magnitude matching holds promise for reducing the personal criterial factor, and at little extra effort over the method of magnitude estimation.

The potential usefulness of magnitude matching is also suggested by the successes of magnitude matching in determining equal-sensation functions within the same modality (Marks, 1974; J. C. Stevens, 1976). Since 1960 , the method has been widely employed intramodally to measure equal-sensation functions in many modalities and thereby to study such sensory phenomena as adaptation, inhibition, temporal summation, spatial summation, locus of stimulation on the sensory surface, and sensory hedonics. (For a review, see Marks, 1974.)

Some may see advantages in the method of magnitude matching besides the ones listed above. Many disagree about the validity of S. S. Stevens' power law. Others agree to the principle but question the validity of the exponents obtained by procedures like magnitude estimation. Attneave (1962), for one, suggested that the psychological magnitudes of numbers are nonlinearly related to the numbers themselves. This suggestion led to the development of a two-stage model for magnitude estimation (e.g., Curtis, Attneave, \& Harrington, 1968; Rule, Curtis, \& Markley, 1970). According to this model, magnitude estimations are the result of two processes-a power transformation from stimulus intensity to internal "sensation" and a power transformation from "sensation" to numerical response. Such a model would imply that the exponents $\beta$ and $\alpha$ of two psychophysical power functions actually represent the products of two power transformations,

$$
\beta=\beta^{*} \cdot C_{1} \text { and } \alpha=\alpha^{*} \cdot C_{2},
$$

where $\beta^{*}$ and $\alpha^{*}$ represent the exponents governing the transformations to sensation and $C_{1}$ and $C_{2}$ represent the exponents governing the transformations to numerical response. If this latter transformation were the same for all sensory and perceptual continua, that is, if $C_{1}=C_{2}$, then the two-stage model would predict the same result in cross-modality matching as would the magnitude estimations themselves, for $\beta^{*} / \alpha^{*}=\beta / \alpha$, as Rule and Curtis (1973) found. Hence, the validity of magnitude matching relies on the consistency, but not on the validity, of magnitude estimation.

The ratios of exponents for any two continua are the same whether one calculates these ratios directly from exponents derived from magnitude estimation 
or first multiplies or divides the exponents by a constant. An analogous point was made by Krantz (1972), and later by $R$. Teghtsoonian (1975) and by Cross (in press); all of them have pointed out that magnitude estimations and cross-modality matches provide an internally consistent system in which the ratio of any pair of exponents is uniquely determined, even if the absolute values of the individual exponents are not. However, as noted above, conventional crossmodality matching is subject to regression, which can make it difficult to determine with precision the exact ratio of exponents: Cross, Tursky, and Lodge (1975) have sought to assess the degree of regression by obtaining cross-modality matches using three continua. Magnitude matching also provides a means to assess the ratio of exponents-or more generally, the relation of one sensory scale to another-while avoiding many of the difficulties encountered in direct crossmodality matching.

\section{AN EXPERIMENTAL TEST OF THE METHOD}

The two continua chosen for study here, loudness and brightness, both have exponents close to .33 when the stimulus levels are expressed in sound energy and luminance (S. S. Stevens, 1975). Hence, the predicted exponent (slope) of the matching functions is about 1.0. The exact size of the predicted exponent is hard to pin down, owing in part to procedural differences from one experiment to another. On the basis of published studies (Hellman \& Zwislocki, 1963; Marks \& J. C. Stevens, 1966; S. S. Stevens, 1955b, 1971), the predicted matching function could have a slope anywhere from 1.0 to 1.2 .

[It should also be pointed out that the exponent and the position of the brightness function depend parametrically on such factors as state of adaptation (J. C. Stevens \& S. S. Stevens, 1963), stimulus size (Mansfield, 1973), and duration (Raab, 1962; J. C. Stevens \& Hall, 1966). The predictions above, about the matching function, are made for a "standard" viewing condition-dark-adapted eyes, target size 1-5 deg, duration 1-3 sec.]

The significance of the intercept (position) of psychophysical functions has often been overlooked in the keen attention devoted to the exponent (slope). Consider, as an example, the kinds of matching functions to be expected from persons suffering nerve deafness and conduction deafness. Compared with the normal function, the nerve-deaf function should have a different slope; the conduction-deaf function should have a normal slope but a different position. Magnitude matching can help to pin down such alterations of position that magnitude estimation would miss.

The prediction about the intercept (absolute position) of the matching function for brightness and loudness is as follows. Given that loudness and brightness are judged on the same scale of sensory magnitude, we can write two equations,

$$
\psi_{\mathrm{L}}=\mathrm{k}_{\mathrm{L}} \phi_{\mathrm{L}}^{\beta}
$$

and

$$
\psi_{\mathrm{B}}=\mathrm{k}_{\mathrm{B}} \phi_{\mathrm{B}}^{\alpha},
$$

where $\psi_{\mathrm{L}}$ is loudness, $\psi_{\mathrm{B}}$ is brightness, $\phi_{\mathrm{L}}$ is sound energy, and $\phi_{B}$ is luminance. Then the full crossmodality matching equation becomes

$$
\phi_{\mathrm{L}}=\mathrm{k} \phi_{\mathrm{B}}{ }^{\alpha / \beta} \text { or } \log \phi_{\mathrm{L}}=\log \mathrm{k}+\alpha / \beta \log \phi_{\mathrm{B}},
$$

where $k=\left(k_{B} / k_{L}\right)^{1 / \beta}$. Since the exponents for loudness and brightness are for practical purpose the same, and since the reference levels for the decibel scales for a $1,000-\mathrm{Hz}$ tone $\left(10^{-16} \mathrm{~W} / \mathrm{cm}^{2}\right)$ and a white light $\left(10^{-10} \mathrm{~L}\right)$ were both selected to lie just below absolute threshold (S. S. Stevens, 1955a), we might expect, to a first approximation, that $x$ number of decibels of tone will match $\mathrm{x}$ number of decibels of light. That is, $\mathrm{k}_{\mathrm{L}}=\mathrm{k}_{\mathrm{B}}$. Hence, since decibel scales are logarithmic, a plot of decibel sound vs. decibel light should yield a straight line of near unit slope and zero intercept.

\section{Method and Procedure}

\section{Apparatus}

Sound. The $1,000-\mathrm{Hz}$ tones were heard through a pair of calibrated earphones (Grason-Stadler TDH-39s, mounted in MX-41/AR cushions). The electronic signal, which was monitored by a frequency counter and precision RMS voltmeter, was passed to the earphones through an attenuator and a switch controlled by the experimenter. Presentation of the tone lasted about $1 \mathrm{sec}$. In the three experiments, the levels ranged from 40 to $95 \mathrm{~dB}$ re $10^{-16} \mathrm{~W} / \mathrm{cm}^{2}$ ("full" range), $40-75 \mathrm{~dB}$ ("low" range), and 60-95 dB ("high" range), in all cases spaced at 5-dB intervals.

Light. The subjects sat in a dark room facing a 3.5-deg circular, white target. Mounted in front of the target was a precision electronic shutter operated by an electronic timer. Mounted to each side of the target were two dim grain-of-wheat lamps that served for fixation. Presentations of the target lasted $1 \mathrm{sec}$.

The light source was a fluorescent lamp (Sylvania Super Deluxe Cool White) mounted outside the booth in front of a hole in the side. To control luminance, calibrated neutral density filters were inserted between the lamp and the hole in the wall; luminance was also varied by means of the voltage to the lamp. In all three experiments, the stimuli were the same-12 luminance levels between 49.8 and $102 \mathrm{~dB}$ re $10^{-10} \mathrm{~L}$. These levels were measured with a precision photometer (Gamma Scientific Model 2000).

\section{- Procedure}

Twelve young paid subjects served in each of the three experiments. The order in which they served was completely counterbalanced. A subject's sessions were spaced by a minimum of 1 day, more typically by 5-10 days.

Upon arrival, the subjects immediately donned red goggles for dark adaptation. Adaptation continued for $18 \mathrm{~min}$ plus another 2 min in the dark booth.

While the subject was adapting, practice was given in the method of magnitude estimation using line lengths from about .5 to $100 \mathrm{~cm}$. 
Then the experimenter gave the following instructions: "In this experiment you will be asked to judge several brightnesses of a light and several loudnesses of a sound. You do this by assigning to each event a number that best seems to match its intensity. You may use large and small numbers, including fractions and decimals, as seem fit, but zero would mean no sensation experienced at all. Try to judge brightness and loudness on a common scale of intensity. Thus if a given light and a given sound seem to have the same intensity they should be given the same number. (This may never happen, but conceivably it could.) Light will always be alternated with sound, but the order of intensities of each will be random. It is very important to assume that the absolute sizes of the numbers and the range of the numbers needed to match the loudnesses may be quite different from those needed to match the brightnesses. In other words, try to make an 'absolute match' of the number and the sensation intensity in each individual presentation. Before each stimulus presentation I will give a ready signal."

As the subject was shown his position in the booth and given the headset, etc., the task was briefly reviewed.

In the experiment using a full auditory range (Condition $\mathrm{F}$ ), there were 12 levels each of tone and light; each stimulus was presented four times in the session. When a low range (Condition $\mathrm{L}$ ) or high range (Condition $\mathrm{H}$ ) was used, each auditory stimulus was presented six times. This arrangement equalized the number of visual and auditory stimuli in all sessions, thereby permitting simple alternation between lights and sounds. For both modalities no stimulus level was repeated until all the others were judged; other than this, the order of levels was random.

In order to control visual adaptation, the interval between successive flashes was about $35 \mathrm{sec}$, except following the two brightest stimuli, when it was a full minute. The total testing time was about $1 / 2 \mathrm{~h}$

\section{Results and Discussion}

An objective procedure, carried out by computer on the magnitude estimates, was used to generate the cross-modality matching functions. The steps of the procedure are outlined in detail in the Appendix. In essence, the procedure is to plot all the geometric means of the estimates and connect adjacent pairs of line segments, as shown in Figure 1. Then as indicated by the horizontal and vertical arrows in that figure, one can determine two stimulus levels, one for brightness, the other for loudness, that match crossmodally. This procedure avoids any assumptions regarding the form (e.g., power functions) of the psychophysical functions relating magnitude estimations to stimulus intensity. This is important because marked departures from power functions sometimes occurred.

Power functions (straight lines in log-log coordinates) are then fitted to the pairs of matching points, and a correlation coefficient computed as a measure of relative goodness of fit.

Each power function is specified by a slope constant and a position constant, that is, an intercept constant. In the present instance, the position of the matching function is specified by a statistic called " $\mathrm{dB}$ 70." This is simply the tone, specified by the number of decibels, that matches $70 \mathrm{~dB}$ light according to the appropriate regression solution. The value $70 \mathrm{~dB}$ was selected because it is near the center of the full auditory range and the visual range. Theoretically, any intercept

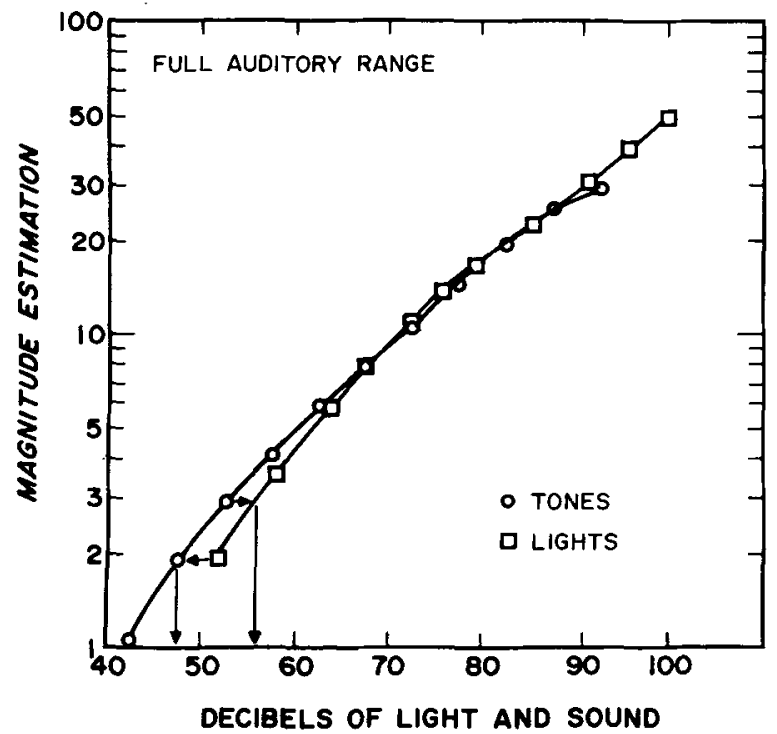

Figure 1. Magnitude estimation of loudness and brightness as functions of decibels of sound $\left(r e 10^{-1 \leqslant} \mathrm{~W} / \mathrm{cm}^{2}\right)$ and decibels of light (re $10^{-10} \mathrm{~L}$ ). The arrows show how two pairs of matching decibel levels are generated graphically. The data are from the experiment using a full auditory range, $40-95 \mathrm{~dB}$.

defined consistently will serve to specify the position of lines in $\log -\log (\mathrm{dB}-\mathrm{dB})$ coordinates. But there is a practical advantage to selecting one that, like $\mathrm{dB} 70$, lies near the middle of the range. Individual psychophysical functions having different slopes tend to intersect, very roughly, in the middle of the range; hence, individual positions defined by an intercept somewhere near the middle will tend to vary far less than when defined at either extreme of the range.

An additional analysis of the data treated the brightness and loudness estimates separately. For each subject, a power function was fitted by the method of least squares; this proved to be useful despite noticeable departures from power functions, especially in the low ranges. The analog to the position constant " $\mathrm{dB} 70$ " is " $\mathrm{dB} 10_{\psi}$ " that is, the number of decibels re $10^{-16} \mathrm{~W} / \mathrm{cm}^{2}$ or decibels re $10^{-10} \mathrm{~L}$ that corresponds to a magnitude estimation of 10 , as determined from each individual's loudness or brightness function. The value $\psi=10$ was chosen because, on the average, it roughly corresponds with $70 \mathrm{~dB}$ sound and light.

\section{Loudness and Brightness Functions and Matching Functions}

The geometric means taken across subjects are plotted in Figures 1-3 for Conditions F, L, and $\mathrm{H}$, showing loudness and brightness in the same coordinates. In most cases, the functions are slightly curved downward and correspondingly depart from simple power functions. Despite this curvature, the overall slopes are, nevertheless, approximately the expected ones-about .3 . 


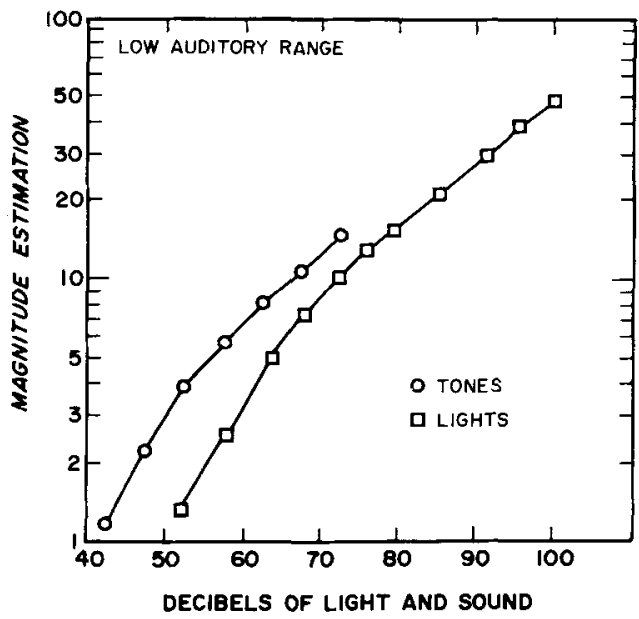

Figure 2. Magnitude estimation under a restricted (low) range of loudness (40-75 dB).

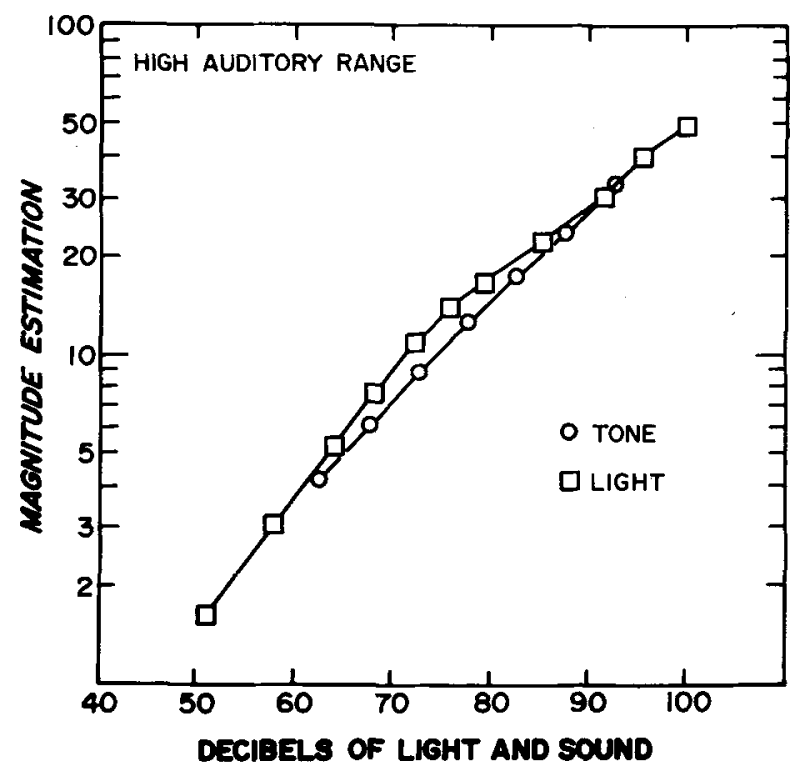

Figure 3. Magnitude estimation under a restricted (high) range of loudness (60-95 dB).

The matching functions derived from Figures 1-3 are shown in Figures 4-6 and Table 1. It is obvious to the eye that these constitute better fits to power functions than the functions from which they derive. The explanation lies, at least in part, in the tendency of a given subject to "warp" the scales for loudness and brightness in much the same way. A clear example is provided by the data of BMA (Condition $\mathrm{L}$ ), as shown in Figure 7 (loudness and brightness functions both curved in the same way) and Figure 8 (no noticeable curvature). Of course, not all the matching functions are as good as those of Figure 8. In order to give a more typical picture, we present, in Figure 9, the data for the median subject, who gave a correlation of .988 .
Individual matching functions occasionally show some curvature, but it is less pronounced than that of the magnitude functions. This can be illustrated objectively by using the correlation coefficient $r$ as a measure of the relative goodness of fit of a power function to each individual's magnitude function and matching function. The top row of Table 2 lists the median $\mathrm{r}$ for each condition computed across subjects. Thus, one advantage of magnitude matching over estimation is to diminish the apparent deviation from the power law shown by some individuals. Correspondingly, of course, any marked curvature in the matching function, provided it can be shown to be reliable, can be taken more seriously as reflecting a true sensory basis, as opposed simply to an idiosyncratic application of the number scale.

The constants of the power matching functions are given in Table 1, both for group and individual data. The group data show that the power function provides an excellent fit (r). They show also that the slope (exponent) of the matching function is as predictedapproximately 1.0 . The position, too, is approximately correct, as shown by the values of $\mathrm{dB} 70$ (close to 70 ). This means that, to a rough approximation, as argued above, decibels of sound $(1,000 \mathrm{~Hz})$ and white light (under standard viewing conditions) match $\mathrm{x} \mathrm{dB}$ per $\mathrm{x} \mathrm{dB}$.

Table 1 shows also that the auditory range affected both the slope and the position. All the differences between mean individual slopes and mean individual position constants are statistically significant by $t$ test ( $p<.05$ to $p<.0001$ ), except the slope difference between Conditions $\mathrm{F}$ and $\mathrm{L}$.

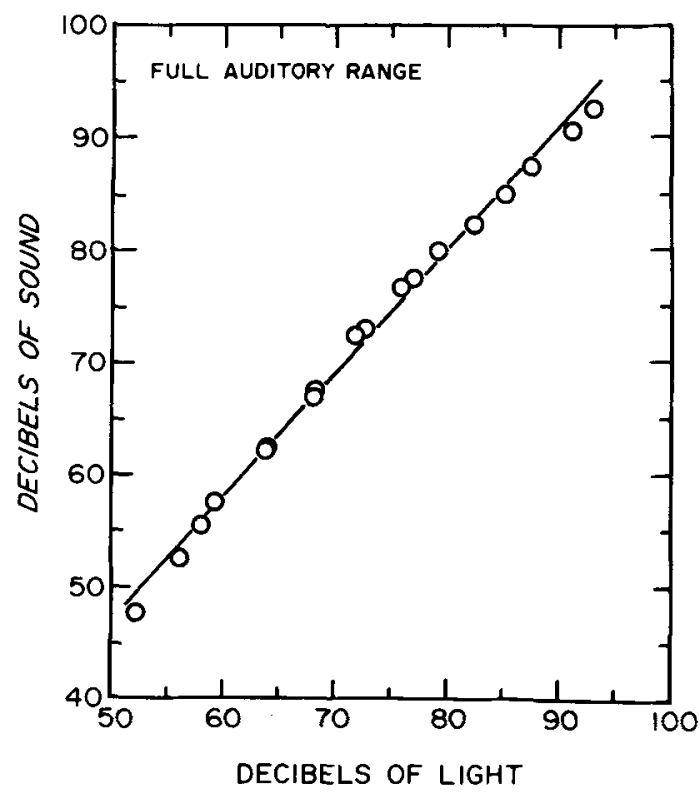

Figure 4. Cross-modality matching function relating light and sound, generated from the data in Figure 1. 


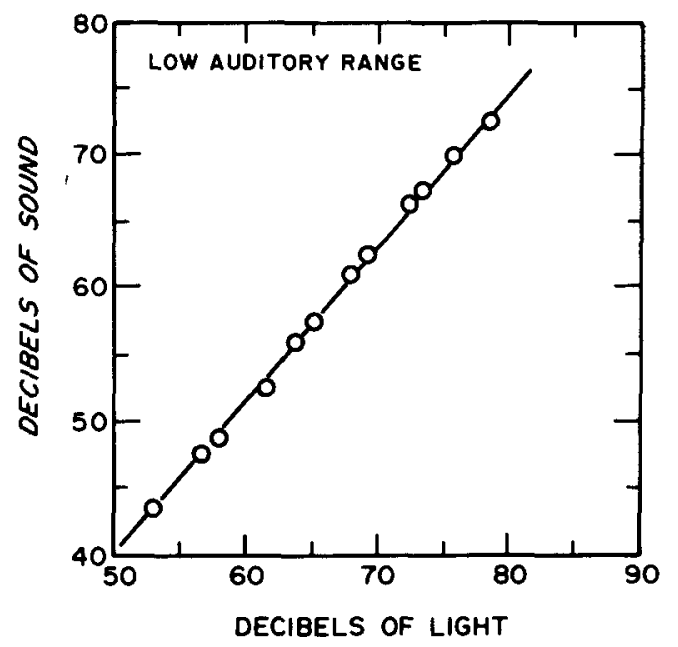

Figure 5. Cross-modality matching function relating light and sound, generated from the data in Figure 2.

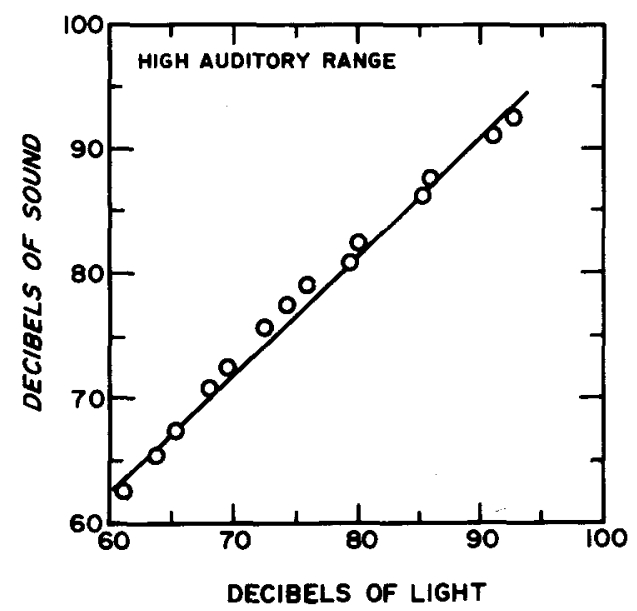

Figure 6. Cross-modality matching function relating light and sound, generated from the data in Figure 3.
Most research on the effects of stimulus range have centered on the slope of magnitude functions, and the general conclusion has been that, up to a point beyond which range no longer matters, the smaller the stimulus range, the steeper the slope of the magnitude estimation function (R. Teghtsoonian, 1973). In the case of magnitude matching in the present study, range had a somewhat more elaborate effect, as might be expected, since both the relative and absolute size of the range varied. Relative to the full range, a low range steepened the slope but lowered the position of the function, while a high range lowered the slope and raised the position. These changes are in line with those reported by Melamed (1970), who showed that range of stimuli to one modality may influence the range of responses on another modality. The effects in the present experiment are real, although minor relative to the radical differences among conditions (in terms of predicted sone values of 1-45, 1-11, and 4-45 sones, respectively). Ideally, the psychological ranges of the two variables should be roughly matched if possible (our intention for Condition F). Practically, when two groups of subjects are to be compared for sensory magnitude, identical stimulus ranges should be used for both groups in order to reduce range effects. For example, if the goal were to learn, say, whether age weakens the sense of smell, then a good procedure would be to match loudness and odorants, using the same range of sound energies and chemical concentrations on groups of subjects of various ages.

It is of interest how early in the test sessions the context effect became evident. The last row of Table 1 shows the constants of matching functions based on the first run of stimuli only, that is, on one estimate per subject rather than four or six. The form, slope, and position are almost identical to those based on all the data.

To determine an average sensory function, a much briefer experiment will do. Our results suggest that

Table 1

Constants of the Magnitude Matching Experiments for Individual Subjects and the Group (Geometric Means)

\begin{tabular}{|c|c|c|c|c|c|c|c|c|c|}
\hline & \multicolumn{3}{|c|}{ Full Range } & \multicolumn{3}{|c|}{ Low Range } & \multicolumn{3}{|c|}{ High Range } \\
\hline & Slope & $\mathbf{r}$ & dB70 & Slope & I & $\mathrm{dB} 70$ & Slope & $r$ & dB 70 \\
\hline R.M. & 1.00 & .986 & 66 & 1.31 & .997 & 64 & .902 & .957 & 75 \\
\hline B.M.U. & 1.08 & .987 & $69^{\circ}$ & 1.10 & .990 & 66 & 1.10 & .979 & 75 \\
\hline A.B. & 1.28 & .988 & 80 & 1.66 & .995 & 72 & 1.20 & .997 & 72 \\
\hline E.M. & 1.15 & .994 & 67 & .812 & .993 & 58 & .878 & .985 & 70 \\
\hline G.J. & .862 & .989 & 67 & 1.02 & .978 & 57 & .889 & .998 & 69 \\
\hline B.M.A. & 1.21 . & .993 & 66 & 1.06 & .999 & 57 & 1.14 & .987 & 63 \\
\hline D.H. & 1.13 & .974 & 69 & 1.67 & .991 & 71 & 1.04 & .973 & 78 \\
\hline R.B. & 1.19 & .946 & 68 & 1.19 & .995 & 65 & .862 & .962 & 77 \\
\hline J.S.* & 1.45 & .988 & 67 & 1.02 & .986 & 66 & 1.09 & .983 & 73 \\
\hline L.B. & .913 & .974 & 67 & 1.22 & .961 & 74 & .748 & .973 & 71 \\
\hline B.O.** & .679 & .998 & 77 & .937 & .999 & 72 & .630 & .984 & 80 \\
\hline M.A. & .984 & .993 & 72 & .994 & .978 & 57 & .794 & .992 & 69 \\
\hline Group Geometric Mean & 1.08 & .997 & 69 & 1.15 & .999 & 63 & .930 & .995 & 72 \\
\hline First Estimates Only & 1.05 & .997 & 70 & 1.27 & .997 & 65 & .950 & .995 & 72 \\
\hline
\end{tabular}

${ }^{*}$ Retest on $F$ yielded slope $=.920, r=.991, d B 70=68 . \quad{ }^{*}$ Retest on $F$ yielded slope $=.952, r=.964, d B 70=74$. 


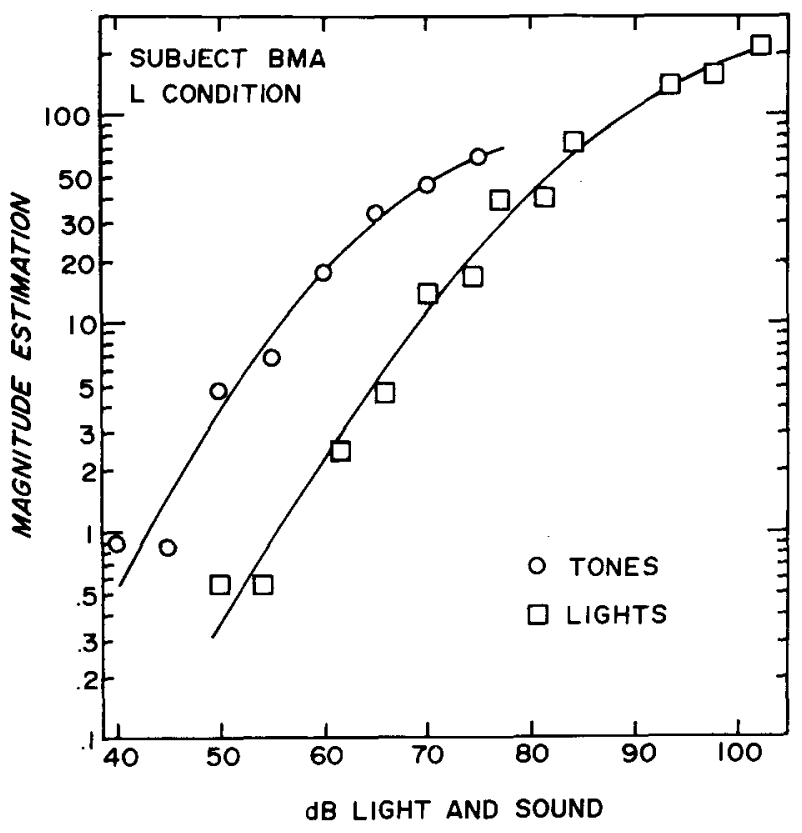

Figure 7. Magnitude estimation of loudness and brightness by Subject B.M.A. under the restricted auditory range (40-75 $\mathrm{dB})$.

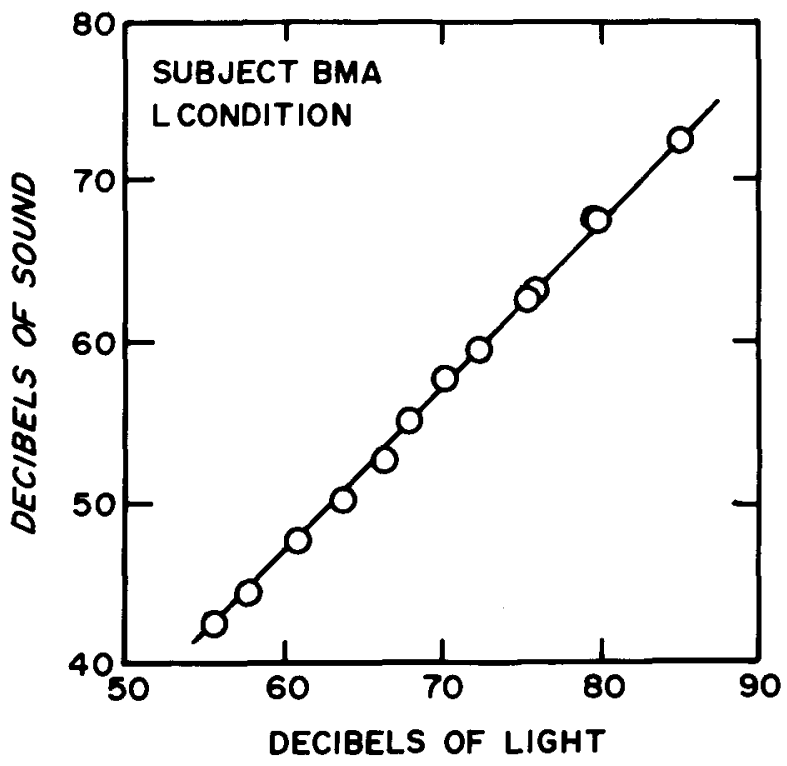

Figure 8. Cross-modality matching function relating light and sound for Subject B.M.A., generated from the data in Figure 7.

about 10 min testing of a dozen subjects should suffice. Given the extra time in a test session, it would also be practical to include stimuli appropriate to other modalities. If, for example, six modalities were judged in a test session, 15 matching functions would be generated. In this way, much information can be gained at relatively little cost.
We turn now to the question of the reliability of the group results. This can be assessed by way of the standard error of the constants of the individual matching functions. These are listed in Table 2, along with the $95 \%$ confidence limits. These show that the group exponents are predictable to within about $\pm 12 \%$ and the position ( $\mathrm{dB} 70$ ) to within about $\pm 3 \mathrm{~dB}$. Is this any better than we would achieve by conventional magnitude estimation experiments?

To help answer this question, we fitted power functions to the individual brightness and loudness estimates, operating on the geometric means of either four (brightness and loudness F) or six estimates (loudness $\mathrm{L}$ and $\mathrm{H}$ ). The constants are listed in Table 2, together with several statistics comparable to those for the magnitude matches. (This turns out to be useful in several ways, even though we know that some of the functions show departures from power functions.) By reference to the $95 \%$ confidence limits, we see that magnitude estimation established the exponent within about $\pm 15 \%$ and the position within about $\pm 18 \mathrm{~dB}$. Thus, we see that the magnitude matching may be somewhat more reliable in slope and is greatly more reliable in position (corresponding values $\pm 12 \%$ and $\pm 3 \mathrm{~dB}$ ). This is to say that magnitude matching permits the determination of absolute magnitude with much greater precision than does magnitude estimation.

Why should this be so? The answer is that a person who gives a high slope for one modality tends to do so for the other, as Ekman et al. (1968), Jones and Marcus (1961), and Jones and Woskow (1962) found when more than one modality was studied within the same test session. This shows up in the form of positive correlations between the exponent of the bright-

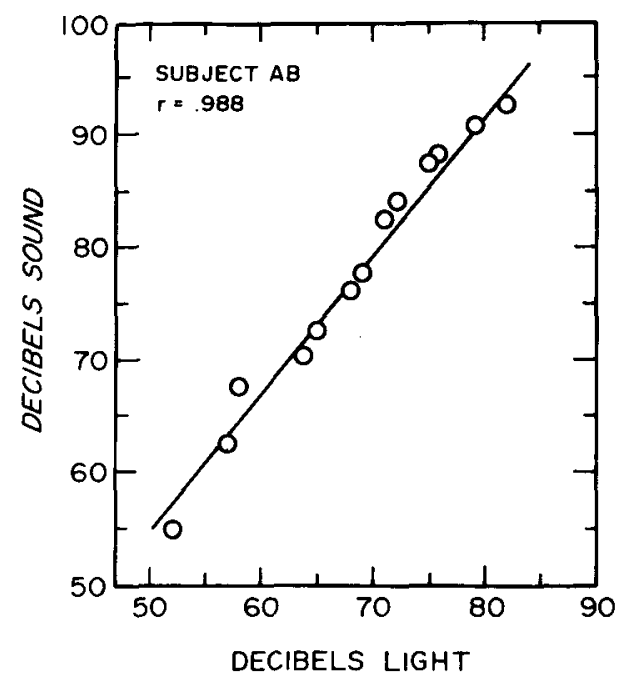

Figure 9. Cross-modality matching for the Subject A.B., whose correlation coefficient equaled the grand median of $r$ for all 36 individual matching functions. 
Table 2

Measures of Central Tendency and Dispersion of the Slopes (Exponents) and Positions of the Individual Cross-Modality Matching Functions and the Individual Magnitude Estimation Functions

\begin{tabular}{|c|c|c|c|c|c|c|c|c|c|}
\hline & \multicolumn{9}{|c|}{ Range } \\
\hline & \multicolumn{3}{|c|}{ Matching Functions } & \multicolumn{3}{|c|}{ Brightness Estimates } & \multicolumn{3}{|c|}{ Loudness Estimates } \\
\hline & Full & Low & High & Full & Low & High & Full & Low & High \\
\hline Median r & .998 & .992 & .984 & .980 & $\begin{array}{r}.980 \\
\text { Slope }\end{array}$ & .978 & .976 & .972 & .989 \\
\hline $\begin{array}{l}\text { Geometric Mean } \\
\text { Geometric Standard Deviation } \\
95 \% \text { Confidence Limits (Group) } \pm \% \\
95 \% \text { Confidence Limits (Individual) } \pm \%\end{array}$ & $\begin{array}{l}1.06 \\
1.22 \\
12.1 \\
47.6\end{array}$ & $\begin{array}{l}1.14 \\
1.24 \\
12.9 \\
52.4\end{array}$ & $\begin{array}{l}.924 \\
1.21 \\
12.0 \\
45.3\end{array}$ & $\begin{array}{l}.275 \\
1.30 \\
16.5 \\
67.7\end{array}$ & $\begin{array}{l}.305 \\
1.27 \\
14.2 \\
59.7\end{array}$ & $\begin{array}{l}.288 \\
1.34 \\
18.5 \\
77.8\end{array}$ & $\begin{array}{l}.286 \\
1.29 \\
16.3 \\
64.4\end{array}$ & $\begin{array}{l}.351 \\
1.33 \\
18.6 \\
74.6\end{array}$ & $\begin{array}{l}.297 \\
1.18 \\
10.0 \\
38.1\end{array}$ \\
\hline & & & & & Position & & & & \\
\hline $\begin{array}{l}\text { Mean } \\
\text { Standard Deviation } \\
95 \% \text { Confidence Limits (Group) } \pm \mathrm{dB} \\
95 \% \text { Confidence Limits (Individual) } \pm \mathrm{dB}\end{array}$ & $\begin{array}{l}69.6 \\
4.52 \\
2.5 \\
8.9\end{array}$ & $\begin{array}{c}64.9 \\
6.46 \\
3.6 \\
12.7\end{array}$ & $\begin{array}{l}72.7 \\
4.70 \\
2.7 \\
9.2\end{array}$ & $\begin{array}{r}75.5 \\
14.8 \\
8.4 \\
29.2\end{array}$ & $\begin{array}{r}75.3 \\
14.8 \\
8.4 \\
28.8\end{array}$ & $\begin{array}{r}74.7 \\
13.5 \\
7.6 \\
26.4\end{array}$ & $\begin{array}{r}70.6 \\
17.4 \\
9.8 \\
34.2\end{array}$ & $\begin{array}{r}65.0 \\
12.8 \\
7.3 \\
25.1\end{array}$ & $\begin{array}{r}74.1 \\
14.7 \\
8.3 \\
28.8\end{array}$ \\
\hline
\end{tabular}

Note-For matching functions, the position constant is number of $\mathrm{dB}$ re $10^{-16} \mathrm{~W} / \mathrm{cm}^{2}$ that match $70 \mathrm{~dB}$ re $10^{-10}$ lambert. For magnitude functions, it is number of $d B$ re $10^{-16} \mathrm{~W} / \mathrm{cm}^{2}$, or $\mathrm{dB}$ re $10^{-10}$ lambert, that correspond to a magnitude estimation of "10." Computation procedures for $95 \%$ confidence limits $\pm \%$ are given in the final paragraph of the Appendix.

ness function and loudness function for each condition $(.72, .72$, and .52 for the $F, L$, and $H$ conditions, respectively, $p<.05$ for $L$ and $H$ ). Thus, slope deviations tend to cancel each other out in the matching function.

The same logic applies to the position constant. A given person may tend to operate on a lower or higher portion of the number scale than average. For example, one person may consistently use the number range .1 to 10 , another 1 to 100 . For the present findings, this shows up in the high correlations between the positions of the individual brightness and loudness functions for each condition $(.98, .90$ and .90 for the $\mathrm{F}, \mathrm{L}$, and $\mathrm{H}$ conditions, $\mathrm{p}<.05$ for all). Like the slope idiosyncrasies, position idiosyncrasies tend to cancel each other in the matching function.

Individual differences in slope and position also tend to persist from one test session to another. This is shown by the correlation coefficients in Table 3, moderate for slope and high for position. This confirms the finding of $\mathbf{M}$. Teghtsoonian and $R$. Teghtsoonian (1971) that individual slopes may be correlated on repeated testing, and extends the finding in a most striking way to include the position of the function.

Table 3

Coefficients of Correlation (r) Between Slopes, and Between Positions (dB 10), Across Experimental Sessions

\begin{tabular}{lccccccc}
\hline & \multicolumn{3}{c}{ Slope } & & \multicolumn{3}{c}{ dB 10 } \\
\cline { 2 - 4 } \cline { 6 - 8 } & F-L & F-H & L-H & F-L & F-H & L-H \\
\hline Brightness & .83 & .84 & .76 & & .96 & .95 & .96 \\
Loudness & .68 & .68 & .40 & & .96 & .93 & .95 \\
\hline
\end{tabular}

Note-All values are significant $(p=.05)$ except for loudness slope $L-H$.
Because of its ability to reduce so many of the sources of variability to which magnitude estimation is subject-variation in form, slope, and position-the method of magnitude matching may come to be the practical method of choice in the evaluation of sensory differences and alteration, whether these be related to sex, age, pathology, drugs (e.g., analgesics), or whatever. Of course, much more testing, in the laboratory and the clinic, will be necessary to establish its usefulness and limitations.

\section{Measuring Individual Sensory Differences}

Of clinical significance is the extent to which a psychophysical method can serve to single out individuals with pathological sensory processes. Although psychophysics has proved immensely useful in this regard (e.g., in clinical audiology) as well as in understanding the basic functional properties of many sensory pathologies, its usefulness has been limited by the lack of control of an individual's personal criterion. For example, many studies of the effects of aging on sensitivity have failed to control for the possible confounding effects of changes of criterion when assessing thresholds with classical psychophysical methods. The recognition of this basic deficiency and a possible remedy for it may be credited to the development of the theory of signal detection.

A similar problem faces the scaling of sensory magnitude by means of magnitude estimation and other related methods. From the very earliest studies involving these methods, it was clear that subjects differ greatly on what they consider to be a given ratio of, say, loudness. Hence, it is unwise to conclude that two subjects truly differ in sensory functioning if, say, their slopes differ by as much as $2: 1$. How large 
a difference can be relied upon and how much improvement might be made by substituting magnitude matching for magnitude estimation? Can a person be made to serve as his or her control to any significant extent?

First-order answers to these questions can be given by reference to the $95 \%$ confidence limits in Table 2 for individual subjects' constants. These teach us that the exponent by magnitude estimation is predictable to within roughly $\pm 65 \%$ and the position to within $\pm 29 \mathrm{~dB}$. This means that only a drastic sensory loss would be picked up reliably by the method and that it would be very easy to be fooled by "false alarms."

For magnitude matching, the comparable figures are $\pm 50 \%$ for the slope and $\pm 10 \mathrm{~dB}$ for the position. This means, for example, that conduction deafness on the order of $15 \mathrm{~dB}$ should be relatively easy to pick up, and the false alarm rate correspondingly rather low (fewer than 1 per 1,000 ), provided the deaf subject's results are compared to a normal group's results obtained under the same stimulus conditions.

In fact, conduction and nerve deafness have been compared cross-modally by Thalmann (1965), who examined 10 patients with one deaf and one normal ear. Five suffered a conduction loss, the other five a sensory-neural loss. The subjects adjusted the amplitude of a $150-\mathrm{Hz}$ vibration on the finger to match the loudness of a $1,000-\mathrm{Hz}$ tone sounded either in the normal or the deaf ear. The median results are shown in Figure 10. (The basic results for individuals were

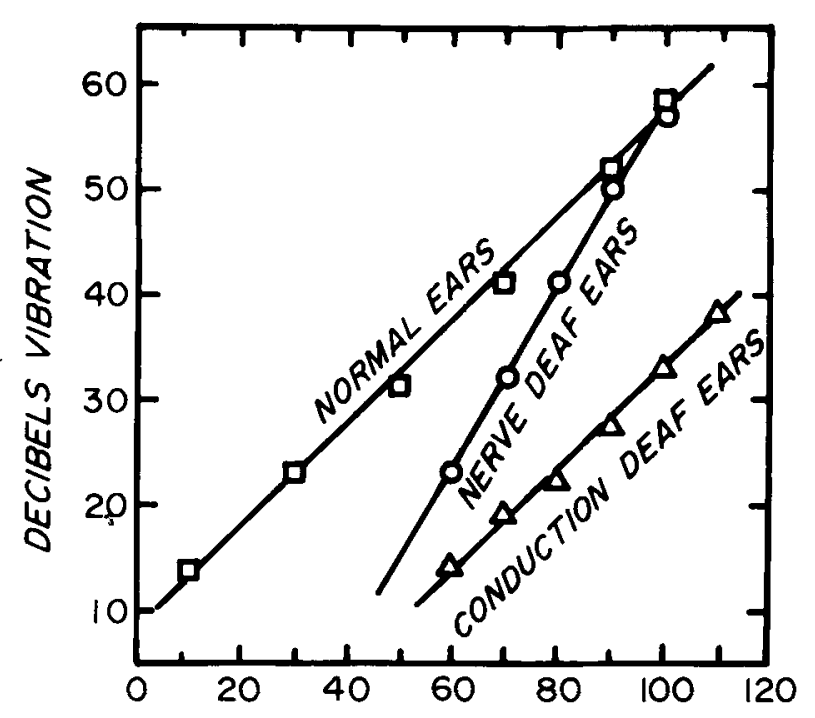

DECIBELS SOUND

Figure 10. Median cross-modality matching of vibration $(150 \mathrm{~Hz})$ to loudness $(1,000 \mathrm{~Hz})$ by 10 deaf subjects examined by Thalmann (1965). Five subjects suffered nerve deafness in one ear; the other five suffered conduction deafness in one ear. The top function shows the matches obtained with the normal ear. (Modified from S. S. Stevens, 1975.) very similar.) Here we see that conduction deafness manifests itself in a drastic shift (about $50 \mathrm{~dB}$ ) in the position of the matching function, with no appreciable change of slope. In contrast, nerve deafness nearly doubled the slope of the matching function and showed a loss of absolute magnitude of about $20 \mathrm{~dB}$ in the vicinity of " $\mathrm{dB} 70$." Losses of such magnitude should be easy to detect in individual patients by the method of magnitude matching. Thalmann also confirmed the validity of the cross-modality matches with direct matches of loudness aroused in the normal and impaired ears.

We have seen that magnitude matching is somewhat more sensitive than magnitude estimation in detecting slope differences and far more sensitive in detecting position differences. In pathologies such as nerve deafness, expected to alter both constants, we may be able to do better by computing the joint probability of obtaining a given deviation of both constants. Such computation will have to take into account that the slope and position constant may be positively, albeit weakly, correlated $(.21, .57$, and .31 for the $F$, $\mathrm{I}$, and $\mathrm{H}$ conditions, respectively, although $\mathrm{p}>.05$ in each case). It is clear, though, that much may be learned by examining the slope and position in conjunction. This finding should prompt the psychophysicist to take more seriously the position of psychophysical functions and to avoid evaluating sensory magnitude solely in terms of the exponent. Both constants carry valuable information. This view underscores the early advice of Hellman and Zwislocki (1963) that the employment of artificial standards by which to make magnitude estimates is essentially to throw away potentially useful information about absolute magnitude.

We can see that neither magnitude estimation nor magnitude matching offers a sensitive measure of individual slopes. Nevertheless, profound losses would show up by magnitude matching. A slope of twice or half the average should occur by chance only 1 in 5,000 tests (assuming our sample of mostly collegeage subjects can be considered representative). Even so, when deviations of such magnitude occur, they should be put to further test. This we did, as examples, in the case of the two most deviant subjects under Condition F, a few weeks after the last previous testing. This time, their results fell close to the average (Table 1), thereby making the point that a subject suspected of abnormality should be retested at long intervals until a clear trend develops.

We conclude that the method of magnitude matching may have use in evaluating individual sensory magnitude as well as average sensory magnitude. At any rate, its chances of success seem greater than those of the method of magnitude estimation per se, and, as we have pointed out, the method retains the basic simplicity of magnitude estimation and takes very little more time and effort to perform. 


\section{REFERENCES}

Attneave, F. Perception and related areas. In S. Koch (Ed.), Psychology: A study of a science (Vol. 4). New York: McGrawHill, 1962.

Cross, D. V. On judgments of magnitude. In B. Wegener (Ed.), Social attitudes and psychophysical measurement. Hillsdale, N.J: Erlbaum, in press.

Cross, D. V., Tursky, B., \& Lodge, M. The role of regression and range effects in determination of the power function for electric shock. Perception \& Psychophysics, 1975, 18, 9-14.

Curtis, D. W., Attneave, F., \& Harrington, T. L. A test of a two-stage model for magnitude estimation. Perception \& Psychophysics, 1968, 3, 25-31.

Ekman, G., Hosman, B., Lindman, R., Ljungberg, L., \& ẢKESSON, C. $\AA$. Individual differences in scaling performance. Perceptual and Motor Skills, 1968, 26, 815-823.

Hellman, R. P., \& Zwislock I, J. J. Monaural loudness function at $1,000 \mathrm{cps}$ and interaural summation. Journal of the Acoustical Society of A merica, 1963, 35, 856-865.

InDow, T., \& Stevens, S. S. Scaling of saturation and hue. Perception \& Psychophysics, 1966, 1, 253-271.

Jones, F. N., \& Marcus, M. J. The subject effect in judgments of subjective magnitude. Journal of Experimental Psychology, $1961,61,40-44$.

Jones, F. N., \& Woskow, M. H. On the relationship between estimates of loudness and pitch. American Journal of Psychology, 1962, 75, 669-671.

KRANTZ, D. H. A theory of magnitude estimation and crossmodality matching. Journal of Mathematical Psychology, 1972, 9, $168-199$.

MANSFiEld, R. J, W, Brightness function: Effect of area and duration. Journal of the Optical Society of America, 1973, 63, 913-920.

Marks, L. E. Sensory processes: The new psychophysics. New York: Academic Press, 1974.

Marks, L. E., \& Stevens, J. C. Individual brightness functions. Perception \& Psychophysics, 1966, 1, 17-24.

MElamed, L. E. The role of response processes in the formation of cross-modality assimilation effects. Perception \& Psychophysics, 1970, 8, 185-188.

RAAB, D. Magnitude estimation of the brightness of brief foveal stimuli. Science, 1962, 135, 42-43.

Rule, S. J., \& Curtis, D. W. Conjoint scaling of subjective number and weight. Journal of Experimental Psychology, 1973, 97, 305-309.

Rule, S. J., Curtis, D. W., \& Markley, R. P. Input and output transformations from magnitude estimation. Journal of Experimental Psychology, 1970, 86, 343-349.

Stevens, J. C. A comparison of ratio scales and category scales for the loudness of white noise and the brightness of white light. PhD dissertation, Harvard University, Cambridge, Mass., 1957.

STEvens, J. C. Equal-sensation functions generated by the method of magnitude estimation. Journal of the Acoustical Society of America, 1976, 59, 473-474.

Stevens, J. C., \& Hall, J. W. Brightness and loudness as functions of stimulus duration. Perception \& Psychophysics, 1966, 1, 319-327.

Stevens, J. C., \& Marks, L. E. Cross-modality matching of brightness and loudness. Proceedings of the National Academy of Sciences, 1965, 54, 407-411.

Stevens, J. C., \& Stevens, S. S. Brightness function: Effects of adaptation. Journal of the Optical Society of America, $1963,53,375-385$.

Stevens, S. S. Decibels of light and sound. Physics Today, $1955,8,12-17$. (a)

STEVENS, S. S. The measurement of loudness. Journal of the Acoustical Society of America, 1955, 27, 815-829. (b)
Stevens, S. S. Perceived level of noise by Mark VII and decibels (E). Journal of the Acoustical Society of America, 1971, $51,575-601$.

Stevens, S. S. Psychophysics: Introduction to its perceptual, neural, and social prospects. New York: Wiley, 1975.

Stevens, S. S., \& Greenbaum, H. B. Regression effect in psychophysical judgment. Perception \& Psychophysics, 1966, $1,439-446$.

Teghtsoonian, M., \& Teghtsoonian. R. How repeatable are Stevens' power law exponents for individual subjects? Perception \& Psychophysics, 1971, 10, 147-149.

TEGHTSOONIAN, R. Range effects in psychophysical scaling and a revision of Stevens' law. American Journal of Psychology, 1973, 86, 3-27.

Teghtsoonian, R. Review of S. S. Stevens, Psychophysics: Introduction to its perceptual, neural, and social prospects. American Journal of Psychology, 1975, 88, 677-718.

Thalmann, R. Cross-modality matching in the study of abnormal loudness functions. Laryngoscope, 1965, 75, 1708-1726.

(Received for publication September 13, 1979; revision accepted November 13, 1979.)

\section{APPENDIX}

In generating the cross-modality matching functions, we wanted to avoid assumptions regarding the form (e.g., power functions) of the psychophysical functions relating magnitude estimations to stimulus intensity. This was important, since marked apparent departures from power functions sometimes occurred. The statistical analysis, performed by computer, which applies alike to individual and group results, involves the following steps:

(1) It computes the geometric means for each level of each stimulus for both loudness and brightness. Geometric means are used because distributions of magnitude estimates are approximately log-normal (J. C. Stevens, 1957).

(2) It takes a running geometric average $(n=2)$ of adjacent geometric means. Strictly speaking, this step is unnecessary, but it is desirable for smoothing some of the inevitable irregularity when the numbers of estimates per stimulus per subject are low. A reanalysis without this step shows that its effect on the group results is negligible; it does have a significant, but small, smoothing effect on the individual functions.

(3) It computes straight-line segments that connect pairs of adjacent geometric means, as adjusted by Step 2 .

(4) Each adjusted geometric mean for loudness is "projected" horizontally onto each line segment for brightness. Its intersection with one or more segments determines the decibel luminances that match the decibel sound energy. (This process is illustrated for simplicity by the arrows of Figure 1.) The process is reversed by projecting the adjusted geometric means for brightness onto the line segments for loudness.

(5) All the pairs of matching points thereby determined can now be plotted as a cross-modality matching function in decibel-decibel coordinates in order to inspect its form. On the assumption of a power function (Equation 3), the computation continues to give the coefficient of correlation $\mathrm{r}$ and two regression solutions (brightness on loudness and loudness on brightness), which are averaged geometrically, since either loudness or brightness can be considered as the (in)dependent variable. 
Additional analysis consists of taking geometric means, standard deviations, and standard errors of the individual slopes. The geometric standard deviation (g.o.), which is defined as the antilog of the standard deviation of the log slopes, represents a ratio rather than an interval; thus, for example a g.o. of 2.0 would mean twice or half the mean. Perfect agreement among subjects (i.e., absence of variability) is indicated by g.o. of 1.0. Geometric standard errors (gSE) must also be interpreted as ratios rather than as intervals.

That geometric rather than arithmetic statistics apply to slopes may seem reasonable to the reader given the way in which the tangent grows from zero to infinity in $90 \mathrm{deg}$. The matter may also be considered another way (see Indow \& Stevens, 1966). Suppose we wish to average a pair of regression slopes: 2.0 and 4.0 . The arithmetic mean equals 3.0, the geometric means equals $\sqrt{2 \times 4}$, or 2.83 . Now, suppose we reverse the coordinates so as to obtain two new regression slopes, .5 and .25 , which average arithmetically to .375. Taking the reciprocal, we see that this corresponds to an agerage of 2.67 , not 3.00. On the other hand, when .5 and .25 are averaged geometrically, $\sqrt{.5 \times .25}$, and the reciprocal taken, we again obtain 2.83 . In other words, geometric, rather than arithmetic, means remain invariant with choice of regression line.

Similarly, $95 \%$ confidence limits can be computed geometrically. For the group data, the formula is: antilog (log geom. mean $\pm 1.96 \mathrm{log}$ geom. S.E.). For the individual data, the formula is: antilog (log geom. mean $\pm 1.96 \mathrm{log}$ geom. o). These confidence limits can also be expressed as percentages above or below the geometric mean by the following formulas:

percentage above $(+\%)=100\left(\frac{\text { upper limit }- \text { geom. mean }}{\text { geom. mean }}\right)$
percentage below $(-\%)=100\left(\frac{\text { lower limit }- \text { geom. mean }}{\text { lower limit }}\right)$

In dealing with the value of $\mathrm{dB} 70$, it is unnecessary to use geometric statistics. Indeed, the decibel notation itself involves a logarithmic transformation so that when decibels are averaged it is akin to geometric averaging. 